\title{
The synaptic Muscle-Specific Kinase (MuSK) Complex: new partners, new functions
}

\author{
Laure Strochlic, Annie Cartaud and Jean Cartaud
}

Biologie Cellulaire des Membranes, Institut Jacques Monod, UMR 7592, CNRS/Universités Paris VI et VII, 2 Place Jussieu, 75251 Paris Cedex 05, France

4210 words with 63 references and 3 figures

Key words: Acetylcholinesterase, acetylcholine receptor clustering, actin cytoskeleton, agrin, congenital myasthenic syndromes, neuromuscular junction, synaptic gene transcription.

Correspondance to: Dr Jean Cartaud, Biologie Cellulaire des Membranes, Institut Jacques Monod, UMR 7592, CNRS/Universités Paris VI et VII, 2 Place Jussieu, 75251 Paris Cedex 05, France; Tel: 331442769 40; E-mail cartaud@ijm.jussieu.fr

\footnotetext{
Abbreviations used in this paper: Abl, Abelson kinases; $\mathrm{AChR}$, acetylcholine receptor; AChE, acetylcholinesterase; APC, Adenomatous Polyposis Coli; ColQ, collagenic tail of AChE; Dvl, Dishevelled; GABP, GA binding-protein; GGT, geranylgeranyltransferase; JNK, c-jun $\mathrm{NH}_{2}$-terminal kinase ; MAGI, membrane-associated guanylate kinase (MAGUK) with inverted domain orientation; MAPK, mitogen-activated protein kinase; MuSK, muscle-
} 
specific kinase; NMJ, neuromuscular junction; NRG, neuregulin-1; PDZ, Postsynaptic density, Disc-large and Zonula occludens.

HUGO gene symbols of human genes involved in NMJ disorders are : acetylcholinesterase, ACHE ; acetylcholine receptor $(\alpha, \beta, \gamma, \delta, \varepsilon$ subunits), $\operatorname{CHRN}(\mathrm{A}-\mathrm{E})$; ColQ, COLQ ; MuSK, MUSK.

Acknowledgments: This work was supported by the Centre National de la Recherche Scientifique, the Universities Paris VI \& Paris VII, and the Association Française contre les Myopathies (AFM). We thank Pr C. Legay for critical reading of the manuscript, members of the laboratory and the two referees for helpful advice. 


\begin{abstract}
:
The muscle-specific kinase MuSK is part of an agrin receptor complex which stimulates tyrosine phosphorylation and drives clustering of acetylcholine receptors (AChRs) in the postsynaptic membrane at the vertebrate neuromuscular junction. MuSK also regulates synaptic gene transcription in subsynaptic nuclei. Over the past few years decisive progress has been made in the identification of MuSK effectors, helping at understanding its function in the formation of the NMJ. Alike AChR, MuSK and several of its partners are the target of mutations responsible for diseases of the NMJ, such as congenital myasthenic syndromes. This minireview will focus on the multiple MuSK effectors so far identified that place MuSK at the center of a multifunctional signaling complex involved in the organization of the NMJ and associated disorders.
\end{abstract}

\title{
Introduction
}

The function of chemical synapses is based on the accumulation of ligand-gated channels or receptors for neurotransmitters in the postsynaptic membrane domain. At the neuromuscular junction (NMJ), nicotinic AChR accumulates in the crests of the postsynaptic membrane with a surface density of $10-20,000 / \mu \mathrm{m}^{2}$ directly beneath the nerve terminal ${ }^{\text {(reviewed }}$ in 1). Extracellular, transmembrane and cytoplasmic proteins, which have structural, adhesive and signaling roles, coaggregate with AChRs in the postsynaptic specialization. The synaptic specialization extends to the basal lamina which is locally enriched in specific isoforms of laminin, in perlecan and acetylcholinesterase (AchE, EC 3.1.1.7) ${ }^{\text {(reviewed in 2) }}$. The synaptic basal lamina also contains two major factors expressed and secreted by motor nerves, agrin ${ }^{(3)}$ and neuregulins (NRG) (reviewed in 4), which are both implicated in synapse formation and maintenance (reviewed in 1, 5). NRG-1 activates ErbB receptors (Receptor Tyrosine Kinase belonging to the EGF receptor family) in the postsynaptic membrane and is thought to trigger transcription of AChR and other synaptic genes. Agrin is a large heparansulfate proteoglycan, first isolated by McMahan and colleagues from Torpedo electric organ, on the basis of AChR aggregating activity in vitro ${ }^{(3)}$. Several agrin isoforms that differ in their capacity to induce AChR clustering are generated by alternative splicing, the most active forms being expressed by motoneurons during embryogenesis precisely during the innervation phase of muscle

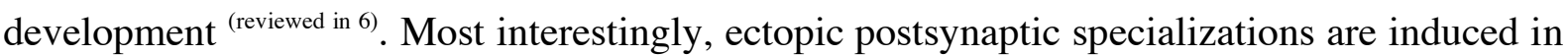
innervated myofibers following injection and expression of plasmids coding for neural agrin (7). The other important component for the differentiation of the NMJ is MuSK, a receptor 
tyrosine kinase specific for muscle cells, ${ }^{(8,9)}$ expressed and concentrated in the postsynaptic membrane in Torpedo electrocyte and in skeletal muscle ${ }^{(8,9,10)}$. Mice deficient for MuSK or agrin have similar phenotypes and lack normal neuromuscular synapses at birth ${ }^{(11,12)}$. These data with previous experiments showing that MuSK is activated by agrin, lead to postulate that $\mathrm{MuSK}$ is a component of an agrin receptor complex. Agrin was initially believed to promote the clustering of AChRs at the NMJ without major effects on transcriptional regulation. However, several studies suggest that agrin could also stimulate the transcription of synaptic genes and that MuSK is required for synapse-specific transcription ${ }^{(7,13-20)}$. MuSKdeficient mice also disclose presynaptic defects in addition to postsynaptic ones, indicating that MuSK activation is also required for presynaptic differentiation. This process involves retrograde signals so far unidentified.

Despite many efforts, the direct physical interaction between MuSK and agrin has so far not been clearly established and the mechanism of activation of MuSK remains obscure. An associated component selectively expressed in skeletal muscle cells, termed MASC (Muscle-Associated Specific Component), has been postulated as coreceptor for agrin to activate MuSK ${ }^{(21)}$. MASC has yet to be identified. Coligands for agrin or posttranslational modifications (glycosylation) either of agrin or of the ectodomain of MuSK could also be envisaged as cofactors for agrin activation of MuSK.

The pleiotropic role of MuSK in synaptic differentiation likely relies on its interaction, either direct or indirect, with a wealth of downstream effectors. In the last few years, several partners of MuSK have been reported, sheding some light onto the mechanisms of action of MuSK. An integrated view of these various mechanisms, taking into account the most recent discoveries in the field, is however still pending. In this review we summarized recent advances in the function of the MuSK complex as a multifunctional platform regulating synapse formation and/or maintenance.

\section{The MuSK signaling pathway involves rapsyn and tyrosine phosphorylations}

Agrin stimulates the dimerization ${ }^{(22)}$ and the kinase activity of MuSK which is essential for clustering of AChRs ${ }^{(23)}$. Signaling triggered from MuSK activation depends upon the phosphorylation of a tyrosine residue (Y553) in the juxtamembrane consensus recognition site (NPXY) for phosphotyrosine binding domain-containing proteins (18, 24) (Figure 1). This phosphotyrosine is required to recruit downstream signaling components involved in many aspects of MuSK signaling, including tyrosine phosphorylation and AChR 
clustering as well as synapse-specific transcription ${ }^{(18)}$. Furthermore, agrin causes rapid activation of Src-related kinases and tyrosine phosphorylation of AChR $\beta$ and $\delta$ subunits ${ }^{(25)}$, the phosphorylation of $\beta$ being required for efficient AChR clustering ${ }^{(26)}$. Tyrosine phosphorylation as well as Src and Fyn kinases are necessary for cluster stabilization ${ }^{(27)}$. However, MuSK kinase domain activation and the resulting phosphorylation of AChR subunits are not sufficient for AChR clustering, indicating that AChR phosphorylation is not the sole trigger of the clustering process ${ }^{(23)}$.

Rapsyn (Receptor-asssociated protein at the synapse), the 43-kDa cytoplasmic peripheral membrane protein associated with AChRs in the postsynaptic membrane ${ }^{\text {(reviewed in 5) }}$ is an essential player in AChR aggregation. Rapsyn-deficient mice die at birth and, like MuSK mutants, lack all-kown features of postsynaptic differentiation, in particular AChR aggregates ${ }^{(28)}$. In these mutants, MuSK normally accumulates at the central region of muscle fibers, indicating that rapsyn is a required intermediate in the pathway that couples MuSK activation to AChR clustering, possibly by recruiting synaptic components to a MuSKcontaining scaffold ${ }^{(29)}$. Unlike the ectodomain of all other receptor tyrosine kinases simply mediating ligand binding, several reports point to an additional role of the ectodomain of MuSK in AChR clustering ${ }^{(18,24,28)}$. Mapping domains on MuSK necessary for its interaction with rapsyn indicate that sequences in and near the fourth IgG-like domain (see Figure 1) are required for association with rapsyn through a putative protein RATL (Rapsyn Associated Transmembrane Linker) to mediate AChR aggregation in quail QT-6 cells ${ }^{(24,29)}$. Examination of agrin-inducing signaling events in rapsyn-deficient myotubes reveals that the agrin-induced MuSK activation occurs normally, whereas MuSK-dependent phosphorylation of AChR (and AChR clustering) does not occur. AChR phosphorylation is thus rapsyn-dependent. Src kinase, the major kinase phosphorylating AChR has been shown to be associated with rapsyn ${ }^{(30,31)}$. Rapsyn may thus function as a scaffolding protein presentating a MuSK-activated Srcrelated kinase to the AChRs.

\section{MuSK triggers the clustering of synaptic proteins via actin cytoskeleton reorganization}

Convergent studies highlight the participation of actin in the process of AChR aggregation following agrin activation of MuSK. Agrin stimulation results in actin polymerization and translocation of the actin regulatory protein cortactin to postsynaptic sites ${ }^{(32)}$. Moreover, latrunculin A, an inhibitor of actin polymerization blocks agrin-induced AChR clustering in myotubes ${ }^{(32)}$. The Rho GTP-ases Rac/Cdc42, two ubiquitous monomeric small 
GTPases regulating actin organization, are required for agrin-elicited clustering of AChRs in myotubes ${ }^{(33)}$. Furthermore, Rac and Rho GTP-ases play distinct but complementary roles in the mechanism of AChR clustering. Rac is necessary for the initial phase of formation of microclusters, and Rho required for their subsequent condensation into full-size AChR clusters $^{(34)}$.

The recent discovery of several MuSK effectors have shed some light on the molecular events downstream of agrin-induced MuSK activation (Figure 2). Interestingly, several of these effectors are involved in actin cytoskeleton organization. In addition to the MuSK kinase activity, a nonreceptor tyrosine kinase (NRTK) activity placed downstream of MuSK is necessary for AChR clustering. Pendergast and collaborators ${ }^{(35)}$ hypothesized a role for Abelson tyrosine kinases (Abl) in synapse formation and reported the requirement of these proteins in agrin-induced AChR clustering in vitro. Abl1 and Abl2 (also known as Abelson and Arg respectively) define a family of NRTK characterized by several domains, including $\mathrm{SH} 2$ and SH3, a tyrosine kinase and a unique C-terminal actin-binding site. Abl kinases regulate actin organization and presynaptic axon guidance ${ }^{(36)}$. Abl kinases are localized to the postsynaptic membrane of the developing NMJ. MuSK and Abl kinases effected reciprocal tyrosine phosphorylation and formed a complex upon agrin engagement. However, the binding site for Abl kinase on the cytoplasmic domain of MuSK remains to be determined. The authors proposed that Abl kinases amplify initial signaling and AChR clustering through tyrosine phosphorylations and stabilize clusters via induction of synaptic actin network ${ }^{(35)}$.

Using the yeast two-hybrid system with the cytoplasmic region of MuSK as a bait, Mei and collaborators ${ }^{(37)}$ identified Dishevelled 1 (Dvl), a scaffolding protein originally discovered in Drosophila that is implicated in the development of coherent arrays of polarized cells via the Wnt signaling pathway. Dvls are cytoplasmic proteins constituted of three domains : DIX (Dishevelled-Axin), PDZ (Postsynaptic density/Disc large/ZO-1) and DEP (Dishevelled-Egl-10-Pleckstrin). The authors demonstrated that Dvl binds to both the juxtamembranous and kinase domains of MuSK through its DEP domain. However, this association is independent of MuSK phosphorylation and the disruption of the MuSK-Dvl interaction has a modest effect ( $\approx$ two-fold decrease) on agrin-induced AChR clustering. Dvl also interacts with the serine/threonine kinase PAK1 (p21-Activated Kinase), which becomes activated in myotubes in response to agrin. PAK1 is an effector of Rac and Cdc42 in actin reorganization. Dvl, by forming a scaffold with MuSK and PAK1, would thus regulate AChR 
clustering mediated by agrin ${ }^{(37)}$. Yet, the confirmation of the role of Dvl in the formation of the NMJ in vivo is awaited.

Another potentially important signaling component in the agrin/MuSK pathway is geranylgeranyltransferase 1 (GGT, EC 2.5.1.10). Double hybrid experiments shown that the $\alpha$ subunit of GGT interacts with the kinase domain of MuSK ${ }^{(38)}$. Agrin causes rapid increase in tyrosine phosphorylation of GGT, and inhibition of either GGT expression or of GGT activity prevents muscle cells from forming AChR clusters in response to agrin. GGT inhibitors prevent agrin-induced activation of Rac1, Cdc42 and PAK1 in myotubes. Moreover, transgenic mice expressing an inactive GGT mutant still able to bind MuSK, exhibit NMJ defects. Strictly, these data indicate that prenylation (a posttranslational modification of proteins catalized by GGT confering membrane targeting) of key components of the MuSK signaling pathway, for example Rac, is necessary for AChR clustering. Since the MuSK/ GGT association is independent of MuSK phosphorylation, a direct demonstration of the role of MuSK/ GGT interaction is still pending.

Evidence for a regulation of AChR clustering by the tumor suppressor APC (Adenomatous Polyposis Coli), an actin-binding protein, has been reported ${ }^{(39)}$. The APC protein has been found in several subcellular compartments of mammalian and drosophila cells, including the cytoplasm, nucleus and adhesive junctions. APC colocalizes with AChRs in the postsynaptic membrane of the NMJ and directly interacts with the $\beta$ subunit of AChRs, helping to localize them to the actin cytoskeleton. Another actin-binding complex, the utrophin glycoprotein complex, is specifically enriched in the postsynaptic membrane ${ }^{\text {(reviewed in }}$ 40). Although not directly involved in the early steps of the AChR clustering process, this complex could play a role in the stabilization of AChR clusters, possibly linking rapsyn to the actin cytoskeleton through dystroglycan ${ }^{(41)}$. One component of the utrophin complex, the phosphoprotein dystrobrevin ${ }^{(42)}$, a substrate for tyrosine kinases, is implicated in the stability of agrin-induced AChR clusters ${ }^{(43)}$.

Finally, the scaffolding protein, MAGI-1c, has been identified by a proteomic approach as a MuSK partner in the postsynaptic membrane in Torpedo electrocytes ${ }^{(44)}$. MAGI-1c ${ }^{(45)}$ is a member of the MAGUK family of scaffolding proteins involved in cell polarity and organization of signal transduction within cellular junctions, excitatory central synapses and the NMJ in Drosophila (reviewed in ${ }^{46)}$. Alike other MAGUKs associated with synapses i.e. the PSD-95/SAP90 and S-SCAM, MAGI-1c is composed of several proteinprotein interaction domains: a Guanylate Kinase (GUK) domain, two WW domains and six 
PDZ domains. Each one of these domains potentially confers specific association with a large pannel of signaling, structural and cytoskeletal elements. MAGI-1c is localized at the Torpedo electrocyte cholinergic synapses and at the adult rat NMJs but was not detected in agrinelicited AChR clusters in myotubes, suggesting that MAGI-1c is not involved in the clustering mechanism. In line with this observation, pull-down experiments revealed that the PDZ 4/5 domains of MAGI-1c interact with the consensus C-terminal PDZ binding site of MuSK (Cartaud et al., unpublished observations), a site that is dispensable for its clustering activity in cultured muscle cells ${ }^{(24)}$. Similarly, PSD-95 family proteins are not required for receptor clustering but are believed to be organizers of excitatory synapses, and are involved in synaptic plasticity ${ }^{\text {(reviewed in } 47)}$. Likewise, MAGI-1c might be involved in the building up of specialized protein complex at the NMJ participating to the terminal differentiation of the NMJ.

\section{MuSK and the regulation of synaptic gene transcription}

Genes encoding AChR subunits, as well as utrophin, rapsyn, AChE and MuSK are selectively transcribed in myofiber nuclei localized beneath the nerve contact. Thus, synapsespecific transcription is believed to participate in concentrating gene products at the NMJ (reviewed in 1, 48) $\mathrm{NRG}^{(4)}$ acting through ErbB tyrosine kinase receptors in the postsynaptic membrane activates synaptic gene transcription via the Mitogen-Activated Protein Kinase (MAPK), c-jun $\mathrm{NH}_{2}$-terminal kinase (JNK) and the Phosphatidyl Inositol-3 Kinase (PI3K) signal transduction pathways (see Figure 3). These pathways converge to the phosphorylation of the Ets transcription factor GA binding-protein (GABP) $\alpha / \beta$. Phosphorylated GABP and JNK/c-jun activate transcription of the synaptic genes via GABP binding to the N-box, a 6-bp

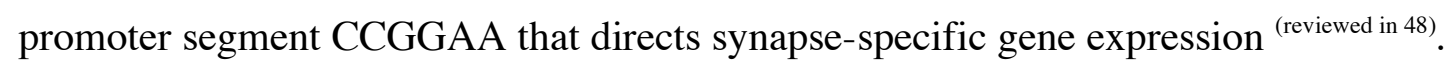

Two negative regulators of the NRG/ErbB pathway, the protein tyrosine phosphatase SHP2 and erbin, have been identified. SHP2 dephosphorylates and inactivates ErbB receptors in the postsynaptic membrane after NRG stimulation and attenuates the NRG-induced expression of the $\varepsilon A C h R$ promoter reporter gene in cultured myotubes ${ }^{(49)}$. Erbin, associates with the C-terminus of ErbB2 through its PDZ domain and down regulates the MAPK signaling cascade stimulated by NRG, through inactivation of Ras ${ }^{(50)}$.

In addition to the NRG/ErbB pathway, several studies pointed to a role for agrin and MuSK in the regulation of synaptic gene transcription. Neural agrin or constitutively active MuSK cluster the ErbB receptors and stimulate transcription of AChR genes ${ }^{(7,51)}$. Recently, 
Brenner and collaborators demonstrated that agrin-induced musk transcription is controlled in part by the NRG/ErbB pathway organized by agrin-MuSK and by a novel shunt path in which MuSK stimulates the activation of Rac and JNK in an independent manner of the NRG/ErbB pathway. Both pathways converge onto the the N-box in the musk and possibly other synaptic gene promoters ${ }^{(15)}$. In fetal muscles, formation of postsynaptic-like sites and targeted gene expression begins to develop in the absence of agrin but depends on MuSK ${ }^{(19,20)}$. Given these data, MuSK appears to be not only involved in AChR clustering but also in the regulation of synaptic genes expression. Interestingly, $\mathrm{Rac} / \mathrm{Cdc} 42$ appear to play a central role in agrin/MuSK signaling conducting both to synaptic gene transcription and AChR clustering (see Figures 2 and 3).

In a systematic search for MuSK partners in situ, Cartaud and collaborators (52) identified the adaptor protein 14-3-3 $\gamma$, using a proteomic approach in purified postsynaptic membrane from Torpedo electrocytes. The 14-3-3 $\gamma$ protein colocalizes with AChRs at the NMJ in rat muscles and coimmunoprecipitates with MuSK. 14-3-3 proteins constitute an emerging family of signaling molecules present in eukaryotic organisms involved in

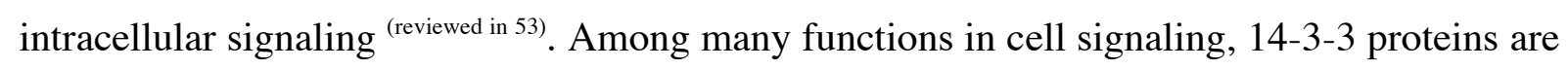
major regulators of the MAPK and PI3K signaling pathways. Overexpression of $14-3-3 \gamma$ in muscle cells specifically represses transcription of several synaptic genes including $\alpha, \delta$ and E-AChR subunits, utrophin (promoter A), rapsyn, and MuSK. The 14-3-3 proteins are known to interact and modulate the activity of Raf- ${ }^{(54)}$. In myotubes in culture, 14-3-3 $\gamma$ inhibits the activation of $\varepsilon$-AChR induced by constitutively active Raf-1, suggesting that it acts downstream of the NRG/ErbB pathway by blocking Raf-1 in an inactive conformation. Moreover, the expression of 14-3-3 $\gamma$ in muscle fibers in vivo causes both the repression of synaptic genes transcription and morphological perturbations of the NMJ. Taken together, these data provide evidence that MuSK may serve as a scaffold to localize and concentrate 14-3-3 $\gamma$ close to the postsynaptic membrane. At this location, 14-3-3 $\gamma$, is prone to interact with signaling molecules of the NRG/ErbB cascade such as Raf-1 (see Figure 3). In many cases, the interaction of 14-3-3 proteins with their various partners is regulated by phosphorylation of serine residues in consensus sequences in the target proteins (reviewed in 55 ). Two such sequences have been identified in the cytoplasmic region of rat MuSK ${ }^{(52)}$. An interesting hypothesis raised by these observations is that a signaling molecule, probably different from agrin, would regulate the MuSK/14-3-3 interaction, thereby regulating the synaptic transcription. 


\section{MuSK localizes asymmetric forms of acetylcholinesterase at the NMJ}

At the NMJ, AChE is mainly present as asymmetric forms in which three tetramers of

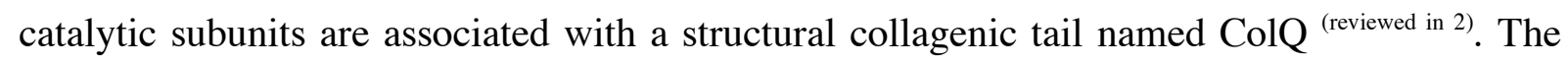
accumulation of the enzyme in the synaptic basal lamina strictly relies on ColQ. This mechanism is mediated by interaction between the collagenous domain of ColQ and perlecan, an heparan sulfate proteoglycan, which itself binds $\alpha$-dystroglycan, a transmembrane glycoprotein of the dystrophin/utrophin complex enriched at the NMJ. In the absence of $\alpha$ dystroglycan ${ }^{(56)}$, perlecan and AChE do not colocalize with AChR. Also, in perlecan null mice, $\mathrm{AChE}$ is absent from the NMJ ${ }^{(57)}$. These data suggest the requirement for the dystrophin/utrophin complex in clustering AChE at synaptic sites. However, recent studies indicate that the C-terminus of ColQ is required for $\mathrm{AChE}$ anchoring at the $\mathrm{NMJ}{ }^{(58)}$, suggesting that another protein might interact with $\mathrm{AChE}$ and contribute the specificity needed for synaptic localization. This protein has recently been identified as MuSK ${ }^{(59)}$. MuSK and ColQ can be crosslinked in situ in Torpedo AChR-rich membranes. Both proteins colocalize and coimmunoprecipitate in COS-7 cells cotransfected with plasmids encoding MuSK and ColQ. Moreover, MuSK is required to form cell surface AChE clusters in cultured myotubes ${ }^{(59)}$. Taken together, these observations suggest that a ternary complex containing ColQ, perlecan and MuSK is required for AChE clustering and demonstrate that MuSK dictates AChE synaptic localization at the NMJ. They also provide a rational molecular basis explaining certain forms of congenital myasthenic syndrome (CMS) associated with mutations in the C-terminal part of ColQ. Surprisingly, in these patients, the asymmetric forms of AChE are normally synthesized, assembled and secreted. Yet, no AChE accumulate

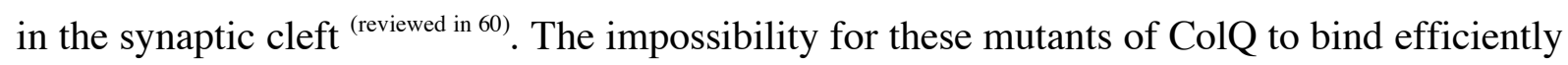
with MuSK at synaptic sites is undoubtedly causal in these disorders.

\section{MuSK : a new target for autoimmune and genetic disorders at the neuromuscular junction}

Because of its importance in NMJ development and function, and alike AChR and other synaptic proteins, MuSK is a possible target for autoimmune and genetic disorders at the NMJ ${ }^{(61,62)}$. Myasthenia gravis is an antibody-mediated autoimmune disease of the NMJ caused, in the majority of patients, by the presence of auto-antibodies to AChR and the consecutive loss of AChRs in the postsynaptic membrane. In $70 \%$ of myasthenia gravis 
patients seronegative for AChR-antibodies, circulating auto-antibodies to MuSK are present (63). These observations define immunologically distinct forms of the disease. The MuSK auto-antibodies were specific for the extracellular domains of MuSK and strongly inhibited MuSK function ${ }^{(63)}$. These antibodies are thus likely involved in the pathogenesis. Other major disorders of the NMJ -the congenital myasthenic syndromes- caused by mutations in key components of the postsynaptic membrane such as AChR subunits and AChE (see above) have been reported ${ }^{\text {(reviewed in } 60)}$. Recently, MuSK has been shown to be the direct target for mutations causing CMS associated with dramatic pre- and postsynaptic structural abnormalities and severe decreases in AChR $\varepsilon$-subunit and MuSK expression ${ }^{(62)}$. Mutations in rapsyn, a downstream effector of MuSK, also caused myasthenic syndromes ${ }^{\text {(review in } 60)}{ }^{\text {. One }}$ could predict that novel mutations in MuSK will be discovered in myasthenic patients, and that MuSK effectors are potential molecular targets for neurological diseases at the NMJ. Understanding the fundamental molecular and cellular bases underlying MuSK function holds the promise of therapeutic approaches for treating these pathologies.

\section{Concluding Remarks}

The increasing knowledge of the MuSK effectors provide a coherent molecular basis to the functional complexity of MuSK, sustaining its pleiotropic function in NMJ formation. In the model proposed (Figure 2), upon stimulation by agrin, MuSK recruits Abl kinases which activate $\mathrm{Rac} / \mathrm{Cdc} 42$ which would in turn stimulate PAK1 and other targets of activated GTPases. Dvl, owing to its interaction with MuSK and PAK1, functions as a scaffold molecule bridging the two proteins to form a signaling complex which leads to local actin reorganization, ultimately conducting to $\mathrm{AChR}$ clustering. In this context an important role for GGT would be to confer membrane localization of Rac/Cdc42, and thus basal Rac/Cdc42 activation. However, the steps in agrin signaling between MuSK activation and actin cytoskeleton anchoring of AChRs via rapsyn are still incompletely characterized.

Other key MuSK partners, 14-3-3 $\gamma$ and ColQ fulfil different dedicated roles in synapse formation and/or maintenance, such as the regulation of synaptic gene expression and the localization of AChE, respectively. How these various molecules operate spatiotemporally and how the multiple signaling pathways are coordinated to orchestrate synaptic differentiation are important issues that should deserve future investigations. In this line, 
MAGI-1c, the PDZ-containing scaffolding molecule associated with the C-terminus of MuSK is potentially central in the organization of the subsynaptic signaling complex. 


\section{References:}

1-Burden SJ. 2002. Building the vertebrate neuromuscular synapse. J.Neurobiol. 53: 501-511.

2-Legay C. 2000. Why so many forms of acetycholinesterase? Microsc. Res. Tech. 49: 56-72.

3-McMahan UJ. 1990. The agrin hypothesis. Cold Spring Harb. Symp. Quant. Biol. Vol. LV: 407-418.

4-Fischbach GD, Rosen KM. 1997. ARIA: a neuromuscular junction neuregulin. Annu. Rev. Neurosci. 20: 429-458.

5-Sanes J, Litchman JW. 2001. Induction, assembly, maturation and maintenance of a postsynaptic apparatus. Nat. Rev. Neurosci. 2: 791-805.

6-Ruegg MA, Bixby JL. 1998. Agrin orchestrates synaptic differentiation at the vertebrate neuromuscular junction. Trends Neurosci. 21: 22-27.

7-Meier T, Hauser DM, Chiquet M, Landmann L, Ruegg MA et al. 1997. Neural agrin induces ectopic postsynaptic specializations in innervated muscle fibers. J. Neurosci. 17: 6534-6544.

8-Jennings CG, Dyer SM, Burden SJ. 1993. Muscle-specific trk-related receptor with a kringle domain defines a distinct class of receptor tyrosine kinases. Proc. Natl. Acad. Sci. USA. 90: 2895-2899.

9-Valenzuela DM, Stitt TN, DiStefano PS, Rojas E, Mattsson K et al. 1995. Receptor tyrosine kinase specific for the skeletal muscle lineage: expression in embryonic muscle, at the neuromuscular junction, and after injury. Neuron 15: 573-584.

10-Bowen DC, Park JS, Bodine S, Stark JL, Valenzuela DM et al. 1998. Localization and regulation of MuSK at the neuromuscular junction. Dev. Biol. 199: 309-319. 
11-DeChiara TM, Bowen DC, Valenzuela DM, Simmons MV, Poueymirou WT et al. 1996. The receptor tyrosine kinase, MuSK, is required for neuromuscular junction formation in vivo. Cell 85: 501-512.

12-Gautam M, Noakes PG, Moscoso L, Rupp F, Scheller RH et al. 1996. Defective neuromuscular synaptogenesis in agrin-deficient mutant mice. Cell 85: 525-535.

13-Jones G, Meier T, Lichtsteiner M, Witzemann V, Sakmann B et al. 1997. Induction by agrin of ectopic and functional postsynaptic-like membrane in innervated muscle. Proc. Natl. Acad. Sci. USA. 94: 2654-2659.

14-Gramolini AO, Burton EA, Tinsley JM, Ferns MJ, Cartaud A et al. 1998. Muscle and neural isoforms of agrin increase utrophin expression in cultured myotubes via a transcriptional regulatory mechanism. J. Biol. Chem. 273: 736-743.

15-Lacazette E, Le Calvez S, Gajendran N, Brenner HR. 2003. A nover pathway for MuSK to induce key genes in neuromuscular synapse formation. J. Cell Biol. 161: 727-736.

16-Cohen I, Rimer M, Lomo T, McMahan UJ. 1997. Agrin-induced postsynaptic-like apparatus in skeletal muscle fibers in vivo. Mol. Cell. Neurosci. 9: 237-253.

17-Jones G, Moore C, Hashemolhosseini S, Brenner HR. 1999. Constitutively active MuSK is clustered in the absence of agrin and induces ectopic postsynaptic-like membranes in skeletal muscle fibers. J. Neurosci. 19: 3376-3383.

18-Herbst R, Burden SJ. 2000. The juxtamembrane region of MuSK has a critical role in agrin-mediated signaling. EMBO J. 19: 67-77.

19-Lin W, Burgess RW, Dominguez B, Pfaff SL, Sanes JR et al. 2001 Distinct roles of nerve and muscle in postsynaptic differentiation of the neuromuscular synapse. Nature 410: 10571064. 
20-Yang X, Arber S, William C, Li L, Tanabe Y et al. 2001. Patterning of muscle acetylcholine receptor gene expression in the absence of motor innervation. Neuron 30: 399410.

21- Glass DJ, Bowen DC, Stitt TN, Radziejewski C, Bruno J et al. 1996. Agrin acts via a MuSK receptor complex. Cell 85: 513-523.

22-Hopf C, Hoch W. 1998. Dimerization of the muscle-specific kinase induces tyrosine phosphorylation of acetylcholine receptors and their aggregation on the surface of myotubes. J. Biol. Chem. 273: 6467-6473.

23-Glass DJ, Apel ED, Shah S, Bowen DC, DeChiara TM et al. 1997. Kinase domain of the muscle-specific receptor tyrosine kinase (MuSK) is sufficient for phosphorylation but not clustering of acetylcholine receptors : required role for the MuSK ectodomain ? Proc. Natl. Acad. Sci. USA 94: 8848-8853.

24-Zhou H, Glass DJ, Yancopoulos GD, Sanes JR. 1999. Distinct domains of MuSK mediate its abilities to induce and to associate with postsynaptic specializations. J. Cell Biol. 146: 1133-1146.

25-Mittaud P, Marangi PA, Herb-Vögtli S, Fuhrer C. 2001. Agrin-induced activation of acetylcholine receptor-bound $\mathrm{Src}$ family kinases requires rapsyn and correlates with acetylcholine receptor clustering. J. Biol. Chem. 276: 14505-14513.

26-Borges LS, Ferns M. 2001. Agrin-induced phosphorylation of the nicotinic acetylcholine receptor regulates cytoskeletal anchoring and clustering. J. Cell Biol. 153: 1-12.

27-Smith CL, Mittaud P, Prescott ED, Fuhrer C, Burden SJ. 2001. Src, Fyn, and Yes are not required for neuromuscular synapse formation but are necessary for stabilization of agrininduced clusters of acetylcholine receptors. J. Neurosci. 21: 3151-3160. 
28-Gautam M, Noakes PG, Mudd J, Nichol M, Chu GC et al. 1995. Failure of postsynaptic specialization to develop at neuromuscular junctions of rapsyn-deficient mice. Nature 377: 232-236.

29-Apel ED, Glass DJ, Moscoso LM, Yancopoulos GD, Sanes JR. 1997. Rapsyn is required for MuSK signaling and recruits synaptic components to a MuSK-containing scaffold. Neuron 18: $623-635$.

30-Fuhrer C, Hall ZW. 1996. Functional interaction of Src family kinases with the acetylcholine receptor in C2 myotubes. J. Biol. Chem. 271: 32474-32481.

31-Qu Z, Apel ED, Doherty CA, Hoffman PW, Merlie JP et al. 1996. The synapse-associated protein rapsyn regulates tyrosine phosphorylation of proteins colocalized at nicotinic acetylcholine receptor clusters. Mol. Cell. Neurosci. 8: 171-184.

32-Dai Z, Luo X, Xie H, Peng HB. 2000. The actin-driven movement and formation of acetylcholine receptors clusters. J. Cell Biol. 150: 1321-1334.

33-Weston C, Yee B, Hod E, Prives J. 2000. Agrin-induced acetylcholine receptor clustering is mediated by the small guanosine triphosphatases Rac and Cdc42. J. Cell Biol. 150: 205212.

34-Weston C, Gordon C, Teressa G, Hod E, Ren XD et al. 2003. Cooperative regulation by Rac and Rho of agrin-induced acetylcholine receptor clustering in muscle cells. J. Biol. Chem. 278: 6450-6455.

35-Finn AJ, Feng G, Pendergast AM. 2003. Postsynaptic requirement for Abl kinases in assembly of the neuromuscular junction. Nat. Neurosci. 7: 717-723.

36-Pendergast AM. 2002. The Abl family kinases: mechanisms of regulation and signaling. Ad. Cancer Res. 85: 51-100.

37-Luo ZG, Wang Q, Zhou JZ, Wang J, Luo Z et al. 2002. Regulation of AChR clustering by Dishevelled interacting with MuSK and PAK 1. Neuron 35: 489-505. 
38-Luo ZG, Je HS, Wang Q, Yang F, Dobbins JC et al. 2003. Implication of geranylgeranyltransferase I in synapse formation. Neuron 40: 703-717.

39-Wang J, Jing Z, Zhang L, Zhou G, Braun J et al. 2003. Regulation of acetylcholine receptor clustering by the tumor suppressor APC. Nat. Neurosci. 6: 1017-1018.

40- Banks GB, Fuhrer C, Adams ME, Froehner SC. 2003. The postsynaptic submembrane machinery at the neuromuscular junction: requirement for rapsyn and the utrophin/dystrophinassociated complex. J Neurocytol. 32:709-726.

41- Cartaud A, Coutant S, Petrucci TC, Cartaud J. 1998 . Evidence for in situ and in vitro association between beta-dystroglycan and the subsynaptic $43 \mathrm{~K}$ rapsyn protein. Consequence for acetylcholine receptor clustering at the synapse.

J. Biol. Chem. 273:11321-11326.

42-Grady RM, Akaaboune M, Cohen AL, Maimone MM, Lichtman JW et al. 2003. Tyrosinephosphorylated and nonphosphorylated isoforms of alpha-dystrobrevin: roles in skeletal muscle and its neuromuscular and myotendinous junctions.

J. Cell Biol. 160:741-752.

43- Grady RM, Zhou H, Cunningham JM, Henry MD, Campbell KP et al. 2000.

Maturation and maintenance of the neuromuscular synapse: genetic evidence for roles of the dystrophin--glycoprotein complex. Neuron 25:279-293.

44-Strochlic L, Cartaud A, Labas V, Hoch W, Rossier J et al. 2001. MAGI-1c : A synaptic MAGUK interacting with MuSK at the vertebrate neuromuscular junction. J.Cell Biol. 153: 1127-1132.

45-Dobrosotskaya I, Guy RK, James GL. 1997. MAGI-1, a membrane-associated guanylate kinase with a unique arrangement of protein-protein interaction domains. J. Biol. Chem. 272: 31589-31597. 
46-Fanning AS, JM Anderson. 1999. Protein modules as organizers of membrane structure. Curr. Opin. Cell Biol. 4: 432-439.

47-Sheng M, Pak DT. 1999. Ligand-gated ion channel interactions with cytoskeletal and signaling proteins. Annu. Rev. Physiol. 62: 755-778.

48-Schaeffer L, de Kerchove d'Exaerde A, Changeux J-P. 2001. Targeting transcription to the neuromuscular synapse. Neuron 31: 15-22.

49-Tanowitz, M., Si, J., Yu, D-H., Feng, G-S. and Mei, L. (1999) Regulation of neuregulinmediated acetylcholine receptor synthesis by protein tyrosine phosphatase SHP2. J. Neurosci. 19: $9426-9435$.

50-Huang YZ, Zang M, Xiong WC, Luo Z, Mei L. 2003. Erbin Suppresses the MAP Kinase Pathway. J. Biol. Chem. 278: 1108-1114.

51-Moore C, Leu M, Müller U, Brenner HR. 2001. Induction of multiple signaling loops by MuSK during neuromuscular synapse formation. Proc. Natl. Acad. Sci. USA. 98: 1465514660.

52-Strochlic L, Cartaud A, Mejat A, Grailhe R, Schaeffer L et al. 2004. 14-3-3 $\gamma$ associates with muscle-specific kinase and regulates synaptic gene transcription at vertebrate neuromuscular synapse. Proc. Natl. Acad. Sci. USA 101: 18189-18194.

53-Van Hemert MJ, de Steensma HY, van Heusden GPH. 2001. 14-3-3 proteins : key regulators of cell division, signalling and apoptosis. BioEssays 23: 936-946.

54-Fu H, Xia K, Pallas DC, Cui C, Conroy K et al. 1994. Interaction of the protein kinase Raf-1 with 14-3-3 proteins. Science 266: 126-129.

55- Muslin AJ, Tanner JW, Allen PM, Shaw AS. 1996. Interaction of 14-3-3 with signaling proteins is mediated by the recognition of phosphoserine. Cell $84: 889-897$. 
56-Jacobson C, Cote PD, Rossi SG, Rotundo RL, Carbonetto S. 2001. The dystroglycan complex is necessary for the stabilization of acetycholine receptor clusters at neuromuscular junctions and formation of the synaptic basement membrane. J. Cell Biol. 152: 435-450.

57-Arikawa-Hirasawa E, Rossi RG, Rotundo RL, Yamada Y. 2002. Absence of acetylcholinesterase at the neuromuscular junction of perlecan-null mice. Nat. Neurosci. 5: 119-123.

58-Kimbell LM, Ohno K, Engel AG, Rotundo RL. 2004. C-terminal and heparin-binding domains of collagenic tail subunit are both essential for anchoring acetylcholinesterase at the synapse. J. Biol. Chem. 279: 10997-1005.

59-Cartaud A, Strochlic L, Guerra M, Blanchard B, Lambergeon M et al. 2004. MuSK is required for anchoring acetylcholinesterase at the neuromuscular junction. J. Cell Biol. 165: 505-515.

60-Engel AG, Ohno K, Sine SM. 2003. Sleuthing molecular targets for neurological diseases at the neuromuscular junction. Nat. Rev. Neurosci. 4: 339-352.

61-Liyanage Y, Hoch W, Beeson D, Vincent A. 2002. The agrin/muscle-specific kinase pathway: new targets for autoimmune and genetic disorders at the neuromuscular junction. Muscle Nerve 25: 4-16.

62-Chevessier F, Faraut B, Ravel-Chapuis A, Richard P, Gaudon K et al. 2004. MUSK, a new target for mutations causing congenital myasthenic syndrome. Hum. Mol. Genet. 24: 3229-3240.

63-Hoch W, McConville J, Helms S, Newsom-Davis J, Melms A et al. 2001. Autoantibodies to the receptor tyrosine kinase MuSK in patients with myasthenia gravis without acetylcholine receptors antibodies. Nat Med. 7: 365-368. 


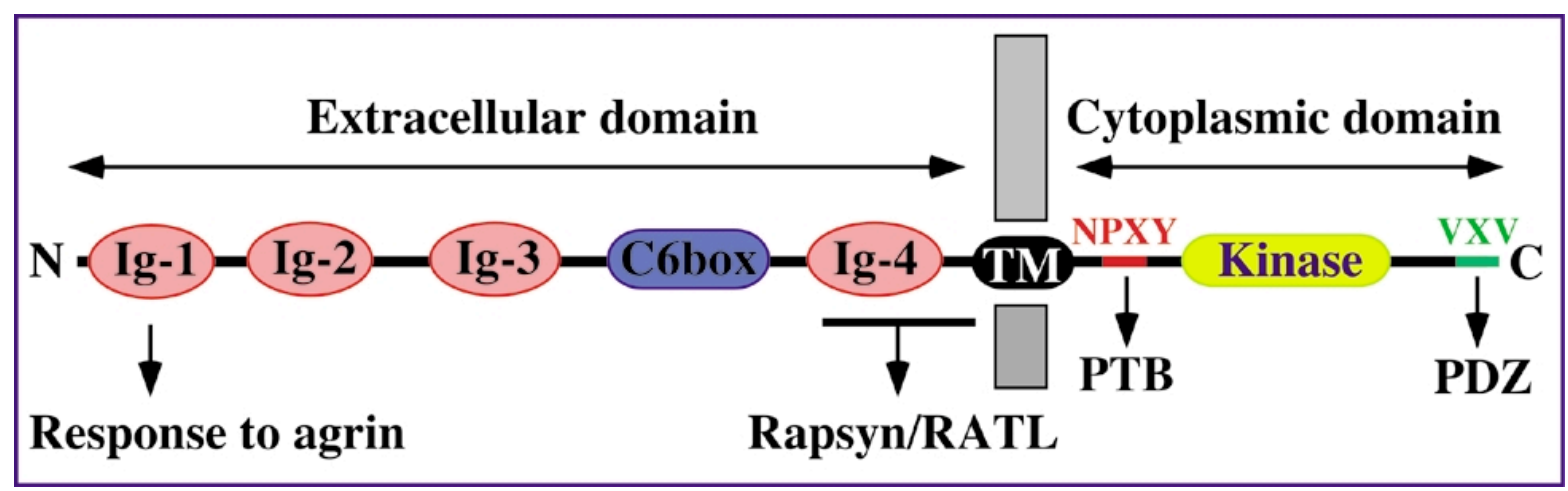

\section{Fig. 1 : Domain organization of the receptor tyrosine kinase MuSK}

The ectodomain of MuSK contained four immunoglobulin-like domains (Ig-1 to Ig-4) and a region containing six phylogenetically conserved cysteine residues (C6 box). The first Ig-like domain is required for agrin responsiveness (interaction with agrin/MASC) whereas the fourth Ig-like domain and adjacent sequences are necessary for rapsyn/RATL interaction (see also Fig. 3). In the cytoplasmic region of MuSK, a recognition site NPXY for phosphotyrosine binding (PTB) domain -containing proteins as well as the ATPbinding/kinase domain are both essential for activity. The C-terminal VXV consensus recognition site for PSD-95/Dlg/ZO-1 like (PDZ) domain -containing proteins is engaged in the binding of MAGI-1c. TM, transmembrane domain. 


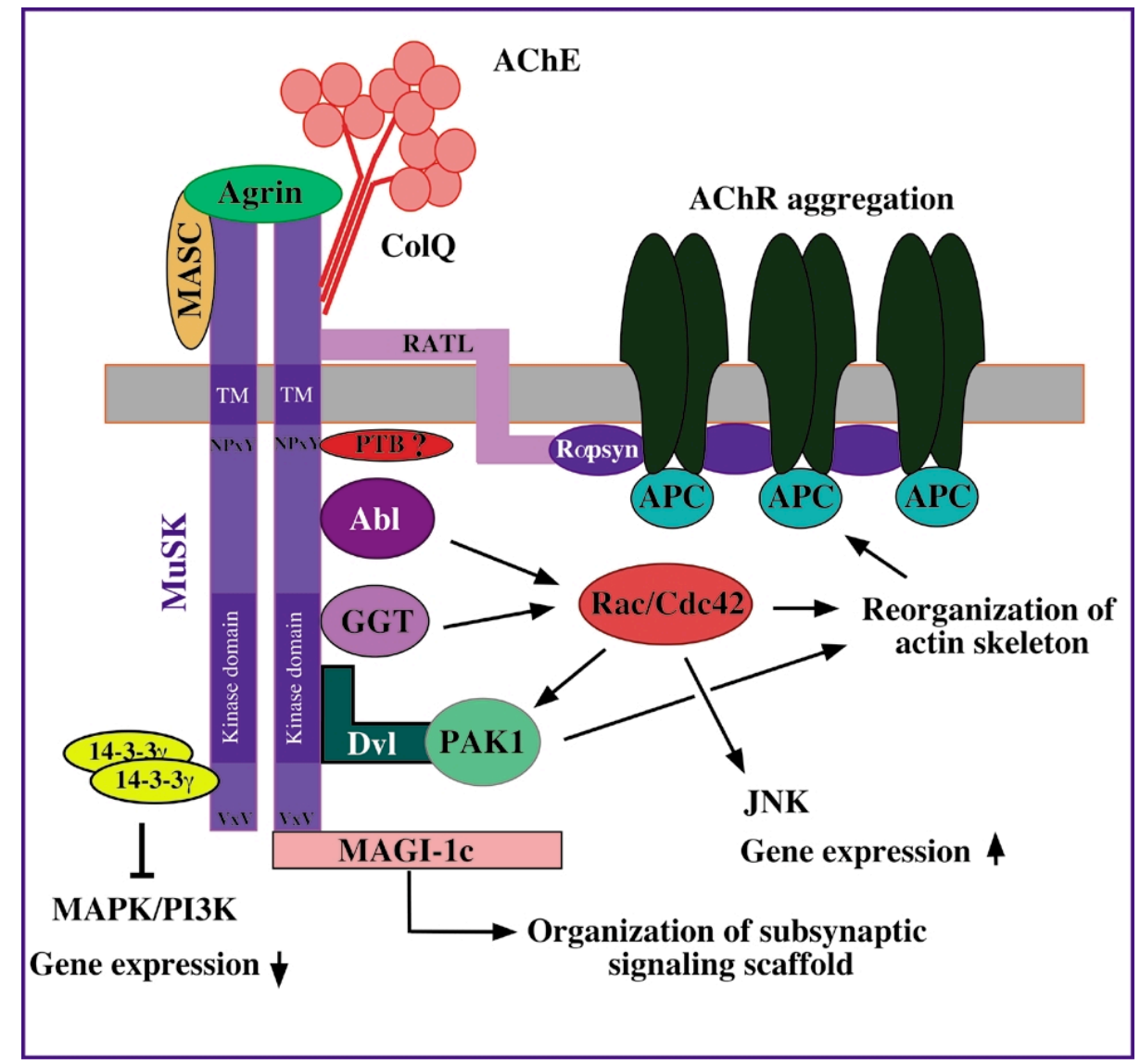

Fig. 2 : Model of the MuSK complex and downstream signaling pathways.

The extracellular domain of MuSK accomodates binding sites for agrin (with the help of the putative muscle-associated specific component, MASC), RATL and ColQ. Cytoplasmic effectors Abl, GGT and Dvl interacting with MuSK activate Rac/Cdc42/PAK1 leading to actin cytoskeleton reorganization and AChR aggregation, likely through APC. The juxtamembrane NPXY motif is required for MuSK signaling through putative PTB domain containing proteins. The scaffolding protein MAGI-1c which binds to the C-terminal consensus PDZ binding site of MuSK (VXV), potentially recruits multiple, yet unidentified, subsynaptic signaling molecules. Finally, the adaptor protein 14-3-3 $\gamma$ regulates synaptic gene expression via inhibition of MAPK/PI3K signaling pathways (see Figure 3). The various molecules are not represented at scale. TM, transmembrane domain. 


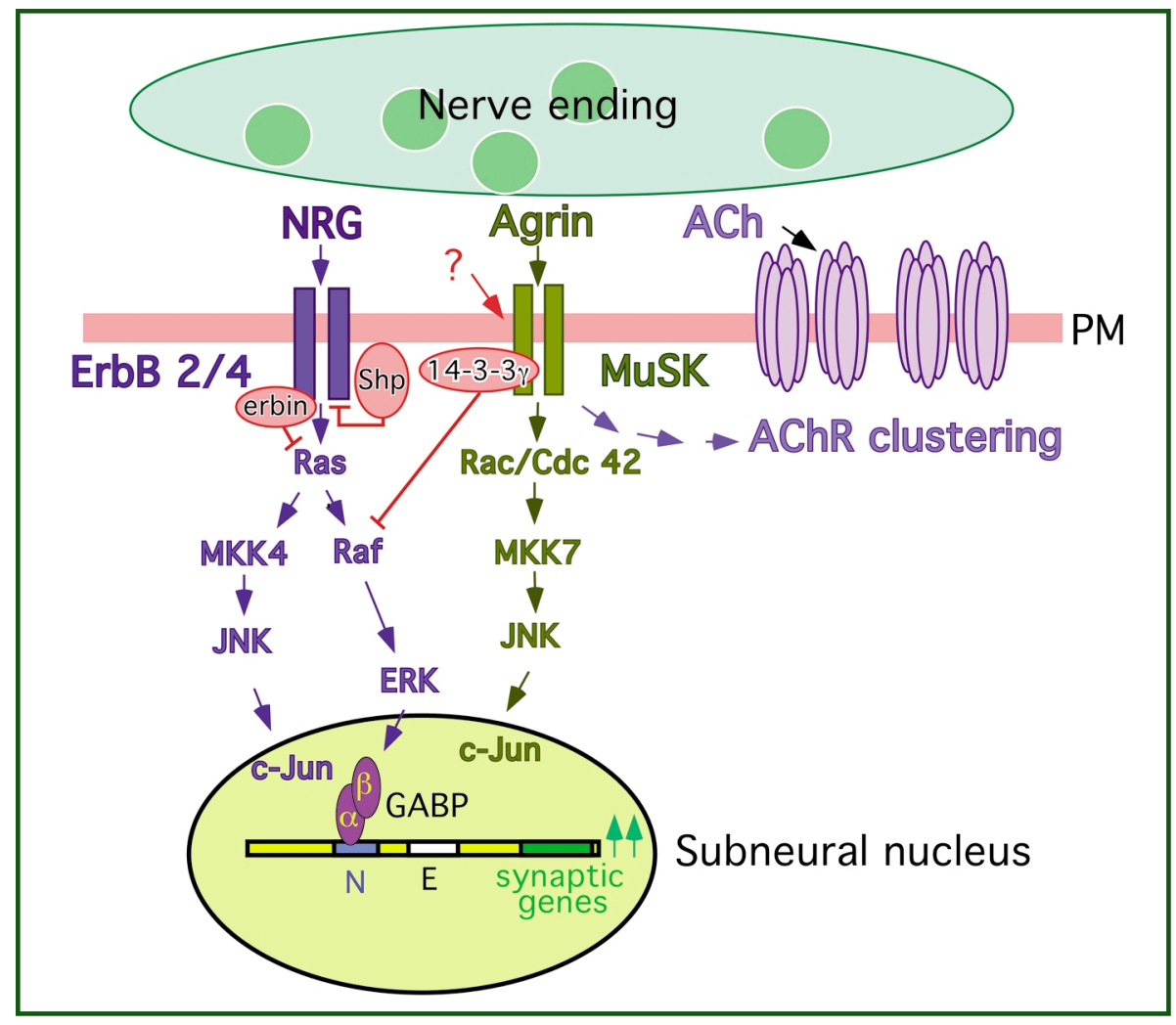

\section{Fig. 3 : Model for transcriptional regulation of synaptic genes at the NMJ}

The tyrosine kinase receptors ErbB 2/4 and MuSK trigger several signaling cascades that cooperate to initiate transcriptional activation of synaptic genes in subsynaptic nuclei. Activation of ErbB receptors by neuregulin (NRG) triggers both the MAPK and JNK pathways via Ras. This lead to the expression of c-Jun and to the phosphorylation of the Etsrelated transcription factor GABP that will activate transcription of synapse-specific genes through binding to the N-box (N). MuSK also induces activation of JNK via Rac/Cdc42, suggesting that the two pathways are connected. Inhibitory factors such as Erbin and SHP2, as well as 14-3-3 $\gamma$ interacting with MuSK, inactivate the ErbB signaling pathway. The question mark indicates a possible regulation of the MuSK/14-3-3 $\gamma$ interaction by a still unknown signal either acting on MuSK or on another signaling molecule. The PI3K pathway is not indicated in the model. (Modified from refs. 15,48 ). 